\title{
Study on the Occupational Personality of Designer and the Training Mode of Design Specialty in the Context of Diversity
}

\author{
Haibo Wu \\ Department of Art and Design, Guangxi Technological College of Machinery and Electricity, Nanning \\ Guangxi, 530007
}

Keywords: Diversified context; Designer; occupational personality type; Design specialty; Talent training mode

\begin{abstract}
Harmony but not Sameness", cultural diversity is the general trend of social development in the future. As a kind of special social occupation, the designer has a profound influence on the development of human civilization. This article is guided by the theory of John Holland on "personality occupation", discusses the professional characteristics of the designer, through analysis of the designer's occupational personality type, to explore the psychological basis of the training mode of design specialty.
\end{abstract}

\section{CLC No.: G40 Document code: A}

\section{Introduction}

Personality has a natural connection with occupation. Thus, sound professional personality has a positive meaning to promote the healthy development of personal career. John Holland, a famous American psychologist, and career counselor, put forward a career interest theory with far-reaching social influence in 1959. He argued that people's interests and personality types were closely linked to their careers and interest was the driving force of people's activities. All the work of professional interests can improve people's subjective initiative, promote people to be actively and happily engaged in the work. And occupational interests and personality have high correlation. Based on this, Holland proposed the "personality - occupation" matching theory, and divided occupational personality into six types: realistic skills (Realistic), explore-research (Investigative), artistic creation (Artistic), social activity (Social), business management (Enterprising) and conventional affairs (Conventional), referred to as RIASEC. (As shown below)

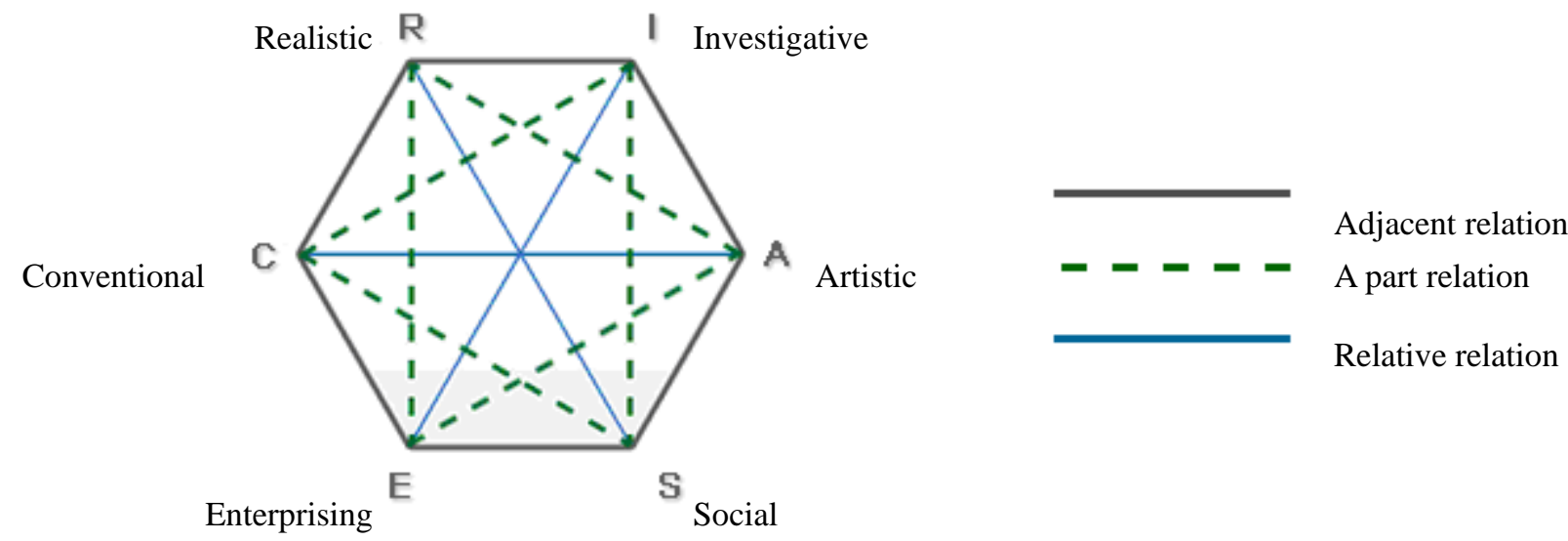

Figure 1. Holland “Personality - Occupation” Matching Theory Hexagon Model

As a specific form of social occupation, designer's work relates to all aspects of human production and life. Especially in the context of diversification, personality needs have gradually become the mainstream of contemporary social consumption concept, prompting designers to solve 
the various problems of target customers through different customized design solutions. The complexity of the designers' working environment determines the diversity of their professional characteristics, so only the designers with complex professional personality can adapt to the increasingly complex and diverse contemporary consumer demand. And the designer's composite professional personality can be cultivated by reforming the existing education model and setting the appropriate curriculum system.

\section{Summary of designer's occupational features}

Designers in ancient China has a specific title, that is, "Baigong"1 . In the Western Zhou Dynasty, it refers to the worker slave, generally including all kinds of handicraftsmen. It is name of construction officer who is in charge of construction and manufacture, and later it's used as the genetic term of a variety of handicraftsmen and handicraft industry. Kaogong Ji recorded "examine the bending and straightening of material to put them in order and distinguish the civil implements", which is the earliest career definition for designers. It describes the comprehensive abilities that designers should have on the shape, material and function of the implements. The writer Liu Zongyuan in Tang Dynasty also made a further discussion on the designer's professional characteristics in his Carpenter Biography. "One chi drawing can include all the specifications, calculate the precise dimensions to construct great buildings without any difference" stresses the designer's rigorous and pragmatic work attitude; "the carpenter dropped his craftsmanship and focused on exercising his intelligence, becoming the person who can master the key construction problems" reflects the overall pattern of thinking of designers; "just like the carpenter who could command the craftsmen, but didn't boast his crafts" highlights the designer's excellent management skills; "the one who can insist his own opinion and make no concession is really a good carpenter" express the professional integrity that the designer should adhere to.

Through the relevant expression of Kaogong $\mathrm{Ji}$ and Carpenter Biography, the evaluation standard for ancient Chinese outstanding designers can be summed up as: superb skills with both ability and political integrity. They not only should have rich professional knowledge and skilled practical skills, but also have pragmatic attitude, excellent management skills, overall mode of thinking and good professional ethics. With the development of history and progress of culture, design has become a means of human creation and communication and has gradually extended to the whole process of human activities. Designers need to work in different areas, and deal with different types of people, as well as solve all kinds of complex problems. In particular, in the contemporary society led by the concept of personalized consumption, designers need to have a more diverse pattern of thinking in order to adapt to social development. Therefore, the comprehensive performance of designer's professional characteristics is reflected on technology, practice, research and management.

1. Technical characteristics: professional skill is the basis of designer's work, a variety of complex practical problems can only be found and solved by becoming an expert in various theoretical knowledge and technical skills.

2. Practical characteristics: the design work is not on paper, and it needs realistic experience of the material process, equipment and tools in practical operation, thus designing effective solution.

3. Research characteristics: the design work is not only to solve the current problems, but also to solve problems in the future. Therefore, designers need to constantly explore new ideas, new materials and new technologies in order to solve the new problems.

4. Management features: design work is the core link of the preparation and implementation of a project. It should be jointly completed by the staff who are under different professional background. Therefore, the designer must have good ability of overall coordination and team management. 


\section{The professional personality of the designer}

Professional personality refers to the psychological characteristics that people should possess as the main body of profession. It is the unique combination of stable attitude and the corresponding behavior way which is suitable for the specific occupation. It is the core part of professional quality of the practitioner. Professional personality is determined by the living environment, education type and the career nature of an individual. Good professional personality can promote the professional concept to become self-conscious behavior. Therefore, sound professional personality is an important psychological basis to adapt to the working environment and complete the task. According to the professional characteristics of the designer, the professional personality manifest on the integration of six types proposed by Holland. It belongs to complex professional personality.

1. Realistic skills type: design is a profession type with strong technicality and practical ability, systematic learning and training is needed to master the professional design knowledge and skills, and solve practical problems in the constant design practice.

2. Explore and research type: design itself is a kind of exploration and research, we cannot use the past thinking to solve future problems. Only through continuous exploration and research, the solution and way can be found to solve new problems.

3. Artistic creation type: design is a combination of art and technology, any new design is a kind of artistic creation, and innovation is the essential requirement of design.

4. Social activities type: the purpose of design is to solve various problems of human society arising in the process of survival and development, provide better life services for people. Extensive participation in various social activities is the inherent requirement of design.

5. Operation management type: design is one of the important link in the market economy. It can not only directly increase the added value of goods, but also is the effective way for contemporary enterprises to create a brand and optimize the management.

6. Conventional business type: design belongs to the service industry. Designers can only effectively solve specific problems based on fully grasping the real needs of target customers and using professional skills and communication strategies flexibly

\section{Designer training model}

Personality has congenital and acquired attributes, so sound professional personality can gradually form through systematic education. According to the inherent requirements of designer's professional characteristics and personality, the training and positioning is mainly concentrated in two aspects: "mind" shaping and "hand-eye" training. "Mind" includes the designer's professional ethics and professional consciousness. It is the designer's soul; "hand-eye" includes the designer's professional knowledge and professional skills, and it is the designer's fundamental. The education of "mind" and "hand-eye" is complementary and consilient whole. Through the theoretical study and social practice of system, the students can achieve the improvement of "hand, eye and heart" in the design thinking and design technology.

Qualified designer not only has good professional knowledge and skills, but also a noble sense of social responsibility. Especially in the commercial design-oriented market economy society, the sense of altruistic humanistic feeling is more needed. Design should promote social and natural harmonious development, rather than mercenary and loss principles. Design, Design Aesthetics and other courses can be set up, from three aspects, including "design essence”, “designer's mission” and "design evaluation". In the enrollment period of new students, develop and establish correct view of professionalism and value for students through the whole teaching and learning links, and in practice, strengthen the students' professional ethics and professional awareness.

Realistic skills type can be trained and improved by setting up a variety of professional design courses integrating theory and practice from the shallower to the deeper and step by step; focus on training students hands-on ability relying on the equipment resources of training center, in practice, let students master new technology, new processes and new materials; exploration and research type 
can explore new design ideas and measures, cultivate students' ability on discovering and solving problems through setting up the course of Design Approach, Design Philosophy, through the research of previous design methodology; artistic creation type cultivate and improve students' artistic appreciation and creativity through setting up the course of Art Appreciation, Creative Thinking; social activities type can train and improve students' social activities ability through setting up Public Relations, Communication and Eloquence etc.; operation management type can train and improve students' management awareness and market awareness through setting up Business Management, Marketing Courses; regular business type can encourage students to participate in a wide range of schools , part-time business activities, cultivate and improve students' ability in social affairs.

\section{Conclusion}

People-oriented is the purpose of contemporary design. Although the designer is responsible for guiding the trend of the times, that does not mean their personal aesthetic view can replace the public aesthetic view. In the contemporary society of cultural pluralism and personalized consumption, the designer's creation does not need to deliberately emphasize his personal will (which is also one of the differences between the designer and artist). The designer's work is only to use their own professional knowledge and skills to skillfully show the social needs in reality, that is, the demand of the public creates the designer's so-called "style". Therefore, designers must have more open and inclusive attitude to integrate into life, learning from the peer and customers, to constantly enrich their own vision and the mind.

Design is a marginal discipline, and a comprehensive scientific category closely linked with different areas, and developed with their results. The purpose of design education in higher vocational education is to cultivate talents of morality and skills in all occupations and promote the development of social economy and culture. Employment-oriented talent training model that takes work process as the carrier must be based on the job characteristics and professional personality, and achieve the new integration of arts and technology, theory and practice, school and society through the establishment of standard curriculum system, and setting up the corresponding professional courses, to develop sound professional sense of personality and skilled professional skills in order to meet the diverse needs of design talents with social development.

\section{Fund Project}

Phased objective of 2015 Guangxi Vocational Education and Teaching Reform Project "Researc $\mathrm{h}$ and Practice on the Segmen-based Project-oriented Teaching Mode of Higher Vocational Art Desi gn Major", Project No.: GXGZJG2015B139

\section{References}

[1] Zeng Ming. Designer's Character - Application Study of Job Matching in the Design Management [D]. Hunan: Hunan University, 2007, (5).

[2] Lu Guofu. Holland Personality Type Theory and College Students Career Counseling [J]. Journal of Guizhou Normal University (Social Science Edition), 2007, (8).

[3] Li Wenxia, Han Weiping. Review on "Personality Type Theory" of Personality and Profession Matching Theory [J]. Journal of Mudanjiang Normal College (Philosophy and Social Sciences Edition), 2010, (2).

[4] Tao Haiying. Ideal Designer - On the Design Idea in Carpenter Biography [J]. Journal of Nanjing Art Institute (Art and Design Edition), 2011, (10).

[5] Liu Jie. Discussion on the Relationship Between Designer Personality Characteristics and Creativity from the Pritzker Prize [J]. Design, 2014, (07). 
[6] Huang Wenjuan, Chen Ronghua. Study on the Model of Designer's Professional Personality Lite racy [J]. "Art Education Research", 2015, (02). 